\title{
Apoptosis in yeast: triggers, pathways, subroutines
}

\author{
D Carmona-Gutierrez ${ }^{1}$, T Eisenberg ${ }^{1}$, S Büttner ${ }^{1}$, C Meisinger ${ }^{2,3}$, G Kroemer ${ }^{4,5,6}$ and F Madeo ${ }^{\star, 1}$
}

A cell's decision to die is controlled by a sophisticated network whose deregulation contributes to the pathogenesis of multiple diseases including neoplastic and neurodegenerative disorders. The finding, more than a decade ago, that baker's yeast (Saccharomyces cerevisiae) can undergo apoptosis uncovered the possibility to investigate this mode of programmed cell death (PCD) in a model organism that combines both technical advantages and a eukaryotic 'cell room.' Since then, numerous exogenous and endogenous triggers have been found to induce yeast apoptosis and multiple yeast orthologs of crucial metazoan apoptotic regulators have been identified and characterized at the molecular level. Such apoptosis-relevant orthologs include proteases such as the yeast caspase as well as several mitochondrial and nuclear proteins that contribute to the execution of apoptosis in a caspase-independent manner. Additionally, physiological scenarios such as aging and failed mating have been discovered to trigger apoptosis in yeast, providing a teleological interpretation of PCD affecting a unicellular organism. Due to its methodological and logistic simplicity, yeast constitutes an ideal model organism that is efficiently helping to decipher the cell death regulatory network of higher organisms, including the switches between apoptotic, autophagic, and necrotic pathways of cellular catabolism. Here, we provide an overview of the current knowledge about the apoptotic subroutine of yeast PCD and its regulation.

Cell Death and Differentiation (2010) 17, 763-773; doi:10.1038/cdd.2009.219; published online 15 January 2010

Over the last decades the budding yeast Saccharomyces cerevisiae has progressively evolved into a preferred research tool in several areas of cell biology. Its easy handling and technical tractability resemble those of bacteria, but are coupled to the functional advantage of yeast being a eukaryote. It has become apparent that, among other cellular processes, the apoptotic core machinery is conserved in yeast to a degree that makes it a suitable model organism to approach pending questions on human apoptosis and its deregulation in the context of neoplasia, neurodegenerative diseases, and aging. At the same time, the existence of a controlled cell death process such as apoptosis invites the consideration that other types of programmed cell death (PCD) processes may be present in yeast, as is the case in mammals (see Box 1). Since the discovery of yeast apoptosis in 1997, ${ }^{1}$ multiple yeast orthologs of crucial mammalian apoptotic proteins have been identified, conserved proteasomal, mitochondrial, and epigenetically regulated cell death pathways have been outlined, and physiological death scenarios have been described. ${ }^{2}$ Assays for apoptotic and/ or necrotic cell death, such as clonogenic determination of viability, accumulation of reactive oxygen species (ROS), DNA fragmentation, exposition of phosphatidylserine, or cell integrity assays, are routinely used in the field of yeast apoptosis. In particular, the use of clonogenic assays allows precise determination of the number of dead versus live yeast cells upon an apoptotic insult (Figure 1).

It is important to underline that it is the combined application of these assays that accurately determines apoptotic death: Similar to the situation in mammalian cells, the evaluation of just one of these methods cannot alone serve to define an observed death phenotype as 'apoptosis'. The terminal dUTP nick-end labeling (TUNEL) test (determining DNA fragmentation), for instance, has been questioned as a proper apoptotic marker in the yeast model. It does, at least under specific cell death conditions, stain positive for single-strand breaks and not for double-strand breaks, which are normally associated with apoptosis in mammalian cells. ${ }^{3}$ In addition, DNA fragmentation has been shown to traverse different phases that all stain positive for the TUNEL test. ${ }^{4}$ It remains to be clarified by correlation with other apoptotic and viability assays whether cells at these various phases (including the early ones) necessarily undergo apoptotic cell death. Still, the TUNEL test has proven to be a reliable apoptotic marker under multiple cell death-inducing conditions, as demonstrated by correspondence with further apoptotic markers., This controversy, therefore, does not invalidate the TUNEL test. However, it certainly underlines the importance of using

\footnotetext{
${ }^{1}$ Institute of Molecular Biosciences, Karl-Franzens University, Graz, Austria; ${ }^{2}$ Institut für Biochemie und Molekularbiologie, ZBMZ, Universität Freiburg, Freiburg, Germany; ${ }^{3}$ Centre for Biological Signalling Studies (bioss), Universität Freiburg, Freiburg, Germany; ${ }^{4}$ INSERM, U848, Villejuif, France; ${ }^{5}$ Institut Gustave Roussy, Villejuif, France and ${ }^{6}$ Université Paris Sud, Paris 11, Villejuif, France

${ }^{*}$ Corresponding author: F Madeo, Institute of Molecular Biosciences (IMB), Karl-Franzens University, Humboldtstrasse 50, Graz 8010, Austria.

Tel: + 43316380 8878; Fax: + 43316380 9898; E-mail: frank.madeo@ uni-graz.at

Keywords: yeast apoptosis; aging; necrosis; unicellular organism; programmed cell death; evolution of cell death

Abbreviations: PCD, programmed cell death; ROS, reactive oxygen species; PTP, permeability transition pore; HDAC, histone deacetylase; $\alpha$-syn, $\alpha$-synuclein; PKA, protein kinase-A; ATG, autophagy-related gene; GAPDH, glyceraldehyde-3-phosphate dehydrogenase; UPR, unfolded protein response; ERAD, endoplasmic reticulum-associated protein degradation; EndoG, endonuclease-G; AIF, apoptosis-inducing factor; TUNEL, terminal dUTP nick-end labeling

Received 18.8.09; revised 07.12.09; accepted 10.12.09; Edited by G Melino; published online 15.1.10
} 
Box 1 Terminology of yeast cell death. The term 'yeast apoptosis' was first used in 1997 to describe specific morphological changes in yeast upon cell death induction. Although the legitimacy of this term has been questioned ever since, several facts have attenuated the initially virulent critique of 'yeast apoptosis.' First, a teleological explanation for the death of a unicellular organism has been provided (see introduction and Figure 2). Second, the expression 'apoptosis' for the death of mammalian cells relies on merely morphological features of cell death, such as the reduction of cellular volume (pyknosis), chromatin condensation, or nuclear fragmentation (karyorrhexis). As recently recommended by the Nomenclature Committee on Cell Death, ${ }^{89}$ the term 'apoptosis' should be applied to cell death events that take place while displaying several of these morphological features. Thus, if this morphological definition is applied beyond the frontiers of the animal kingdom, it is undeniable that yeast cells do frequently succumb to apoptosis.

In this context, it is indispensable to emphasize that PCD describes a highly heterogeneous process regulated by distinct (although sometimes partially overlapping) subroutines. In mammals, these specific mechanisms include programmed necrosis (not to be confused with accidental cell death or 'classical necrosis'), apoptosis, and autophagic cell death, according to their etiology or morphology. The discovery of yeast apoptosis and its high degree of functional conservation allows the investigation of this kind of PCD in yeast, and also opens doors to the concept that other forms of PCD may regulate yeast cell death. In fact, programmed necrosis has recently been described as an active regulatory mechanism during chronological aging in yeast. ${ }^{7}$ Regarding the scientific correctness and the applicability of the yeast cell death system to higher eukaryotes, it is, hence, imperative to precisely define the phenotypic results and distinguish between possible different cell death modalities, especially in certain scenarios where separate executioner cascades may be activated simultaneously. For the accuracy of the term 'yeast apoptosis,' it is, thus, crucial to keep in mind that apoptosis is not a synonym for PCD, but rather an individual subroutine within the PCD machinery.

different assays in each specific scenario tested to carefully determine the actual mode of death. In addition to the classical assays, new markers for necrosis, such as nuclear release of the yeast HMGB1 (Nhp6Ap), a non-histone chromatin protein, or complete disintegration of subcellular structures determined by electron microscopy, are currently being established and will help to better distinguish between apoptotic and necrotic death. ${ }^{7}$

Although being the most extensively studied one, $S$. cerevisiae is not the only unicellular organism that can exhibit typical markers of apoptosis. In fact, apoptosis has been described for other model organisms such as the yeast Schizosaccharomyces pombe or the slime mold Dictyostelium discoideum, as well as for a number of pathogenic fungi and protozoan parasites. ${ }^{2}$ Thus, the teleological question arises why a unicellular organism should have developed and conserved a suicide program during evolution in a context in which the demise of a single cell can be viewed as the death of the organism. However, this is only true from a classical point of view: in fact, conceiving why yeast apoptosis follows the same physiological purpose known for multicellular organisms, namely eliminating superfluous cells, demands a conceptual change. Yeast populations should not be interpreted just as a group of partitioned unicellular organisms that do not communicate among each other, but rather as a multicellular community of interacting individuals. Under certain circumstances, the death of a single cell might be beneficial for the whole population, thus promoting the survival of the clone. Several physiological scenarios in which altruistic death of single cells promotes survival of the population as a whole support this idea. ${ }^{8}$ For example, during aging or development of multicellular colonies, where death of older and damaged cells preserves vanishing resources; thereby, dead cells release nutrients, differentiation molecules, and as yet unidentified pro-survival factors that can be metabolized and stimulate the survival of younger and fitter cells. ${ }^{4,9-11}$ In addition, increased ROS production accompanying enhanced cell death raises the probability of somatic mutations in the rest of the population and, thus, generation of genetic variants that can adapt to continuously changing conditions. In fact, regrowth of a subpopulation of betteradapted mutants has been shown to partly depend on the superoxide levels. ${ }^{9}$ Coupling cell death control to the process of aging limits single cell longevity, thus avoiding the maintenance of ancient genetic variants within the population and hindering genetic conservatism. During failed mating, mating-type pheromones trigger cell death to clean the culture from infertile or otherwise damaged haploid cells, thus guaranteeing the adaptive benefit of the diploid state, which allows meiotic recombination and the resulting genetic diversity. ${ }^{12}$ However, apoptosis can also occur during meiosis of diploid cells, which might ensure that only genetic recombinants that are adapted to their surroundings survive. ${ }^{13,14}$ Apoptosis resulting from these different physiological scenarios (Figure 2) ameliorates the long-term survival of the population and, consequently, increases the chances of spreading of the clone.

Yeast apoptosis and the aforementioned teleological explanation for its existence underscore the evolutional advantage of such an orchestrated suicide program. Indeed, a glance at the conservation of this process reveals that various forms of PCD are known to be present in a wide range of phylogenetically diverging branches of the evolutionary tree. This functional conservation reaches back to worms such as Caenorhabditis elegans (emerged 700 million years ago), ${ }^{15}$ the plant kingdom ( 1 billion years), ${ }^{16}$ unicellular eukaryotes (1-2 billion years), ${ }^{2}$ and even bacteria ( 4 billion years). ${ }^{17}$ Thus, evolution seems to persistently select cellular self-destruction mechanisms, which over time have been harnessed and refined with the growing complexity of organisms. Death regulation as an integral part of cellular organization has, hence, contributed to the propagation of life in the course of natural history. This has not only happened via cooperation of single cells in multicellular communities, but also through intercellular and inter-clonal competition. For instance, single yeast cells can undergo cooperative altruistic cell death for the benefit of their population (see above), but can also be subject to the hijack of their own cell death program by competing with yeast strains that produce and secrete virus-encoded killer toxins, ${ }^{18}$ possibly in their fight for nutrients. Of note, such a hijack of yeast cell death programs has been shown to occur as a form of plant and animal defense against pathogenic fungi. ${ }^{19,20}$ Figure 2 summarizes different natural scenarios activating yeast apoptosis, which may lead to either long-term survival of the clone or to externally triggered death as a consequence of antifungal defense or inter-fungal competition.

This paper primarily attempts to summarize our current knowledge of the different triggers of yeast apoptosis as well as of the molecular players involved in the apoptotic 


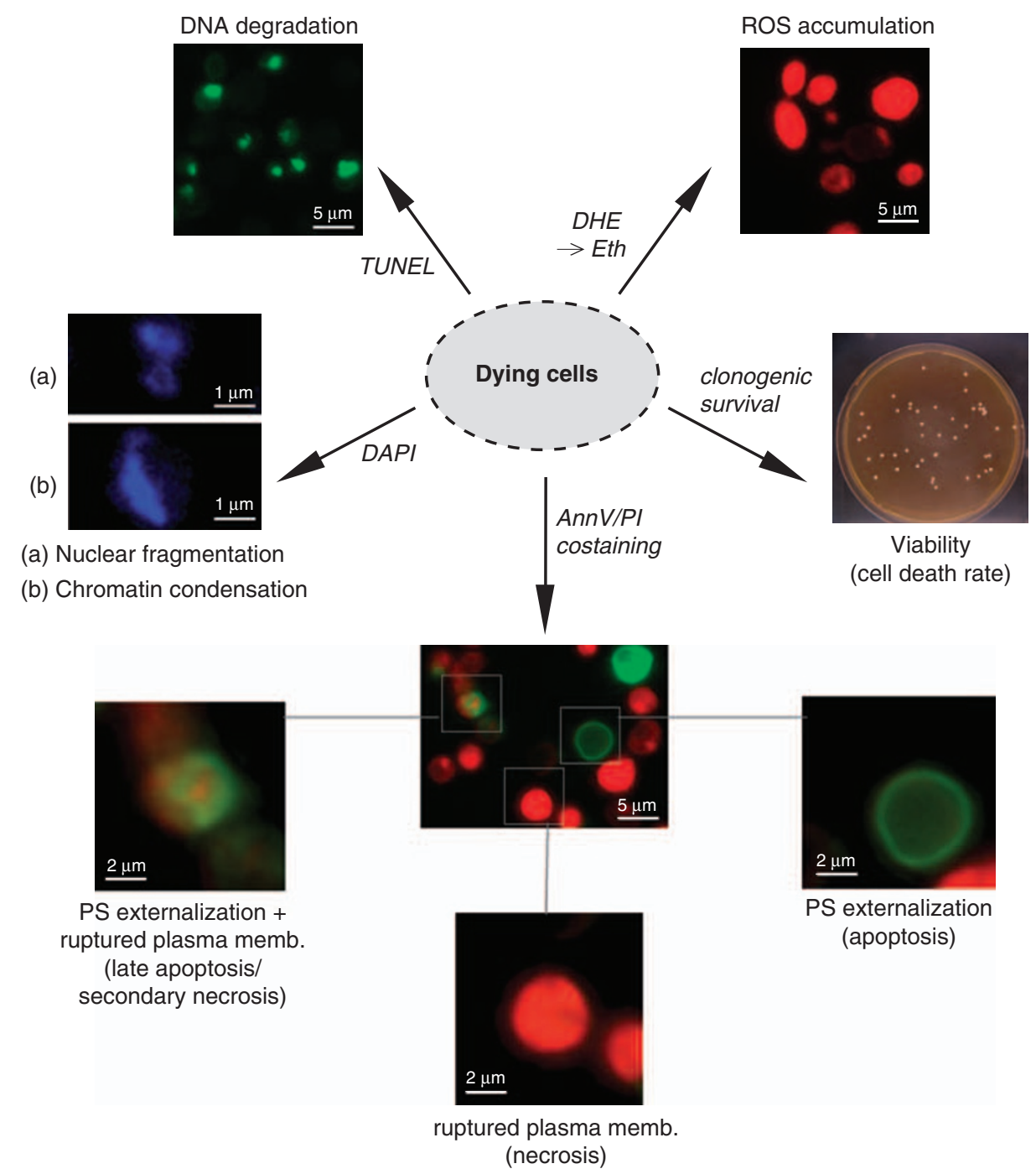

Figure 1 Assays routinely used in the field of yeast PCD. Co-staining of annexin-V (AnnV) and propidium iodide (PI) allows discrimination between early apoptotic cells exhibiting phosphatidylserine (PS) externalization $\left(\mathrm{AnnV}^{+}, \mathrm{Pl}^{-}\right)$, cells showing ruptured plasma membrane indicative of primary necrosis $\left(\mathrm{AnnV}^{-}, \mathrm{Pl}^{+}\right)$, and late apoptotic/ secondary necrotic cells, which show both PS exposition and membrane permeability $\left(\mathrm{AnnV}^{+}, \mathrm{Pl}^{+}\right)$. While apoptotic DNA fragmentation is measured using the TUNEL test, generation of ROS is usually assessed using dihydroethidium (DHE), where the superoxide-driven conversion of non-fluorescent DHE into fluorescent ethidium (DHE $\rightarrow$ Eth) can be monitored in an automated assay. In all cases, stained cells are commonly visualized with a microscope and quantified by cytofluorometry. In addition, nuclear fragmentation and chromatin condensation can be observed upon DAPI staining. An important advantage of yeast is the possibility to easily evaluate actual cell death rates by using clonogenic assays, in which a fixed amount of cells is plated and their ability to form a colony is determined. As only viable cells will form a colony, precise quantification of the amount of dead versus living cells is possible

self-execution of yeast cells. At the same time it sheds light on the mounting evidence pointing toward the existence of other PCD subroutines such as programmed necrosis.

\section{Triggering Yeast Apoptosis}

Numerous stimuli can induce yeast apoptosis. Such stimuli can be provided externally in the form of chemical or physical stress, via heterologous expression of human proapoptotic proteins (exogenous triggers) or by the yeast cells themselves, as part of lethal signal transduction pathways (endogenous triggers).
The exogenous triggers hydrogen peroxide and acetic acid. The first proof of ROS being key regulators of yeast apoptosis (see below) arose from experiments involving treatment of $S$. cerevisiae with low doses of hydrogen peroxide $\left(\mathrm{H}_{2} \mathrm{O}_{2}\right)$. Whereas high doses of $\mathrm{H}_{2} \mathrm{O}_{2}$ lead to a necrotic phenotype, low doses induce apoptosis. ${ }^{21}$ Several molecular factors are involved in this process, including the yeast caspase $Y C A 1^{22}$ and the apoptosis-inducing factor-1 (AIF1). ${ }^{6}$ Consistently, knockout of either YCA1 or AIF1 increases resistance to $\mathrm{H}_{2} \mathrm{O}_{2}$. Similarly, deletion of $\mathrm{RHO}$, a small GTPase with a Ras-like effector region, leads to reduced cell death upon $\mathrm{H}_{2} \mathrm{O}_{2}$ induction. Interestingly, Rho5p interacts with the thioredoxin reductase Trr1p, a key component 


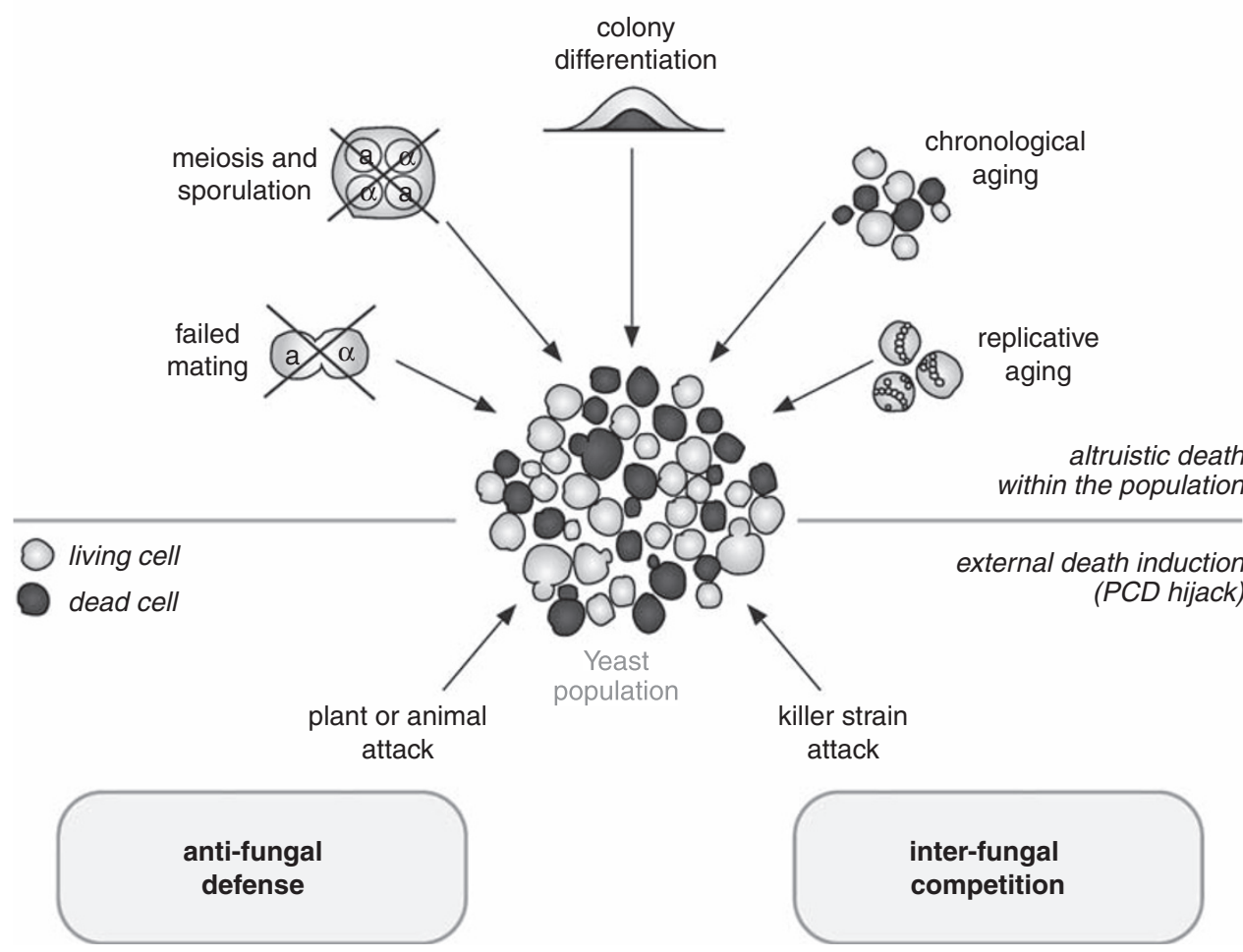

Figure 2 Physiological scenarios of yeast apoptosis. A wild-type yeast population promotes its own long-term survival and spreading of the clone by eliminating infertile or otherwise damaged cells (failed mating), or genetic recombinants not adapted to the surroundings (meiosis and sporulation). In addition, death of old cells within the colony center feeds the young cells at the colony margin (colony differentiation). The death of chronologically old cells preserves resources, releases nutrients, and allows adaptive regrowth (chronological aging), whereas replicatively old cells die for the good of young cells, which inherit the undamaged cellular material upon cellular division (replicative aging). However, death in the population may also be triggered by toxins from either non-clonal enemy strains in competition for nutrients (killer strain attack), or higher eukaryotes in their defense against pathogenic fungi (plant or animal attack). In these cases of external cell death induction, the endogenous apoptotic machinery is hijacked

of the cytoplasmic thioredoxin antioxidant system. ${ }^{23}$ Thus, one may suspect Rho5p, being a transmitter of $\mathrm{H}_{2} \mathrm{O}_{2}$-induced cell death signals, and Trr1p, being a major element in the regulation of redox homeostasis, to act in an antagonistic manner under oxidative stress.

Acetic acid represents another compound commonly used to induce yeast apoptosis. ${ }^{24,25}$ Treatment of yeast cells with acetic acid leads to mitochondrial cytochrome $c$ release $\mathrm{s}^{26}$ and depends on the presence of the yeast orthologs of adenine nucleotide translocator, a protein involved in the proapoptotic mitochondrial outer membrane permeabilization through the so-called permeability transition pore (PTP). ${ }^{27}$ Disruption of cytochrome $c$ partially prevents acetic acid-induced cell death, which is linked to enhanced mitochondrial membrane potential and loss of cytochrome $c$ oxidase activity. ${ }^{26}$ Consistently, $\rho^{\circ}$ cells, which lack mitochondrial DNA and, hence, are respiration-deficient, display resistance against acetic acid-induced cell death. ${ }^{26}$

Recent studies have elucidated further details on acetic acid-induced apoptosis, including requirement of temporary activation of the proteasome ${ }^{28}$ which suggests the existence of a cross-talk between the antioxidant and the proteolytic systems. A recent report sheds light on a possible role of acetic acid in the regulation of chronological aging via extrinsic acidification of the culture medium. Consistently, long-lived yeast mutants such as sch9 or ras2 are highly resistant to cell death induction by acetic acid. ${ }^{29}$ Interestingly, the signaling of acetic acid-induced apoptosis is linked to amino-acid metabolism as well as the TOR pathway, which both connect the nutritional situation of the cell to the aging process (see below). ${ }^{30}$

Further external triggers: from metals to drugs. A variety of additional agents have been reported to induce an apoptotic phenotype in yeast. These include ethanol, hypochlorous acid $(\mathrm{HOCl})$, high salt $(\mathrm{NaCl})$, UV irradiation, or heat stress. ${ }^{2}$ Several compounds, which normally constitute nutrients or oligo-elements, can trigger apoptosis when they are applied at supraphysiological, toxic concentrations. This applies to glucose, sorbitol (hyper-osmotic stress), copper, manganese, and iron. ${ }^{2}$ Interestingly, ironinduced yeast death might involve Isc1p, a neutral sphingomyelinase that can be activated by cardiolipin and is involved in the biosynthesis of ceramide. ${ }^{31}$ In fact, 
mounting evidence points toward an important role of ceramide in yeast cell death, ${ }^{32}$ exemplifying lipotoxicity as an emerging research area. ${ }^{33}$ For instance, only recently was exogenous administration of unsaturated fatty acids in the absence of neutral lipids characterized to mediate apoptosis in yeast in a manner depending on the degree of unsaturation. ${ }^{34}$ The possibility to study lipotoxic death in yeast allows exploring the mechanisms governing cellular lethality upon lipid imbalance, which commonly occurs in association with obesity and probably influences many of the effects, such as diabetes and atherosclerosis, that are linked to it.

Different heavy metals have also been related to yeast cell death. The lethal action of cadmium depends on the yeast caspase Yca1p and glutathione synthesis. ${ }^{35}$ Another metal associated with yeast apoptosis is calcium. Its cytosolic concentration rises following amiodarone (an antiarrhythmic drug) or $\alpha$-factor-induced apoptosis, eventually leading to mitochondrial fragmentation in a process that depends on the yeast suicide protein Ysp1p. ${ }^{36}$ An additional link between yeast death and $\mathrm{Ca}^{2+}$ is mediated by the calcineurin/calmodulin system, which is connected to ER stress regulation. ${ }^{37}$

In nature, the functional potential of yeast undergoing apoptosis is hijacked for different purposes (see Figure 2). For instance, the toxins ricin and osmotin are produced by plants to trigger apoptosis in pathogenic fungi. Osmotin functions by activating the Ras pathway of apoptosis via the receptorlike polypeptide Pho36p, a homolog of the mammalian adiponectin receptor. ${ }^{38}$ Amphibians have also developed mechanisms to prevent the invasion of fungal pathogens: amphibian-derived peptides from the dermaseptin family can induce Aif1p-dependent but Yca1p-independent yeast apoptosis. $^{19}$ Furthermore, so-called yeast 'killer strains' express virus-encoded toxins that induce Yca1p-mediated apoptosis in other yeast cells that are present in the same niche and compete for resources. ${ }^{18}$

Various pharmacological agents can induce yeast apoptosis. This applies to aspirin; the anti-mitotic, microtubule-stabilizing agent paclitaxel; the antitumor synthetic lipid edelfosine; the antileukemic agent arsenic, whose action depends on the mitochondrial translocase TIM18; and the antibiotic bleomycin, which is also used in cancer therapy. ${ }^{39}$ Moreover, low doses of valproic acid, an inhibitor of histone deacetylases (HDACs) in mammalian cells, which displays antitumor activity and is extensively used to treat neuropsychiatric disorders, induces YCA1-dependent yeast apoptosis, ${ }^{35}$ which is prevented upon deletion of the HDAC SIR2. ${ }^{40}$ Treatment of yeast cells with amiodarone also triggers yeast cell death, in this case via the mitochondrial pathway and preceded by a $\mathrm{Ca}^{2+}$ boost. ${ }^{36}$ The transcriptional response of yeast cells to amiodarone treatment includes two signatures: one that resembles a $\mathrm{Ca}^{2+}$ stress response that is accompanied by downregulation of genes involved in all stages of cell-cycle control, and another that is $\mathrm{Ca}^{2+}$-independent and affects nutrient-responsive genes. ${ }^{41}$ Diverse other antitumor agents, such as 5-fluorouracil or coumarin, shown to be cytotoxic for yeast, ${ }^{39}$ need further investigation regarding the induced mode of cell death in order to establish a possible link to apoptosis. Finally, a series of antifungal drugs have been related to yeast apoptosis, including the antifungal antibiotic pradimicin or the metal cation chelator ciclopirox olamine. ${ }^{39}$
Pheromones: natural triggers of yeast apoptosis. Exposure of haploid cells to low doses of their corresponding mating pheromone causes apoptosis in the context of unsuccessful mating or when suitable mating partners are absent. Interaction of the pheromone with its receptor (Ste2p or Ste3p) results in activation of the MAP kinase-signaling cascade (which involves the key kinase Ste20p), subsequent increase of intracellular $\mathrm{Ca}^{2+}$, and a rise in mitochondrial activity, followed by cytochrome $c$ release and apoptosis. ${ }^{12,36}$ In contrast, death caused by elevated concentrations of pheromone lacks certain hallmarks of apoptosis. ${ }^{42}$ Of note, sporulation following meiosis of diploid cells is coupled to apoptosis as well. ${ }^{14}$ Interestingly, both apoptosis and meiosis in yeast have been demonstrated to involve $\mathrm{H} 2 \mathrm{~B}$ phosphorylation. ${ }^{13}$ It is, thus, conceivable that such phosphorylation marks in the chromatin structure regulate the cell's decision to succumb to cell death or enter the sporulation process.

Heterologous expression of human proapoptotic proteins. Heterologous expression of the human key apoptotic inducer Bax in yeast leads to apoptotic cell death accompanied by cytochrome $c$ release. ${ }^{43}$ Conversely, heterologous expression of $\mathrm{Bcl}-2$ or $\mathrm{Bcl}-\mathrm{xL}$ prevents Bax-induced lethality and improves resistance to $\mathrm{H}_{2} \mathrm{O}_{2}$ and acetic acid. ${ }^{43} \mathrm{Bcl}-2$ expression can also reduce mutation frequency and extend the chronological life span in yeast. ${ }^{44}$ These data suggest that specific, regulated action of Bax rather than an uncontrolled effect mediated by the toxic action of an overexpressed xenogenic protein (such as unspecific pore formation), induces yeast apoptosis. However, the contribution of the yeast PTP complex or mitochondrial respiration to Bax-induced cell death still remains a matter of debate. ${ }^{43}$

It has been shown that heterologous expression of $\alpha$-synuclein ( $\alpha$-syn), an intracellular trigger of Parkinson's disease, has detrimental effects on yeast cells and induces apoptosis. $^{45}$ During chronological aging (the prolonged culture of yeast cells in a confined environment), $\alpha$-syn decreases the life span and increases the frequency of apoptotic and necrotic events. ${ }^{46}$ Intriguingly, this effect is abrogated in cells lacking mitochondrial DNA ( $\rho^{\circ}$ cells), revealing a strict requirement of functional mitochondria for $\alpha$-syn toxicity. ${ }^{46}$ These results underscore the possibility to use yeast as a model organism for $\alpha$-synucleinopathies (e.g. Parkinson's Disease) and similar pathologies such as polyglutamine diseases (e.g. Huntington's Disease). ${ }^{47,48}$

Endogenous triggers: mutations and aging. Defects in several cellular processes such as $\mathrm{N}$-glycosylation, chromatid cohesion, mRNA stability, and ubiquitination can trigger cell death in yeast. ${ }^{2,49-51}$ Moreover, DNA damage and replication failure can stimulate the activation of yeast cell death programs; ${ }^{52}$ oxygen metabolism and ROS generation are thereby major causes of DNA damages. In yeast, several players involved in DNA-damage-regulated apoptosis have been found, including tRNA methyltransferase- 9 , which acts as a tRNA modifier that positively regulates the expression levels of major DNA-damage-response proteins, ${ }^{53}$ or the peroxiredoxin Tsa1p, identified as a key peroxidase able to suppress genome instability. ${ }^{54}$ 
Genomic instability, which contributes to aging in all eukaryotes, is intimately related to replication stress, which in yeast affects both chronological and replicative aging. Yeast replicative aging defines the number of divisions an individual mother cell undergoes before dying and provides a model for the aging of proliferating cells, especially asymmetrically dividing stem cells. Division of yeast cells occurs in an asymmetrical manner (including the asymmetrical distribution of mitochondria), resulting in one daughter cell, which acquires undamaged cellular material and, hence, is reset to a status of youth, and one mother cell, which is characterized by a remaining bud scar, and retains the damaged contents of the progenitor cell, thus growing one division step older. After several division cycles, the replicatively aged mother cell eventually undergoes cell death that is accompanied by typical apoptotic markers such as ROS overproduction, phosphatidylserine externalization, and DNA fragmentation, ${ }^{11}$ thus eliminating damaged cellular material from the population.

Chronological aging of yeast serves as a model for aging of human post-mitotic cells. The chronological life span represents the time a culture remains viable in the post-diauxic and stationary phase. Under these conditions of restricted nutrient availability, unfit cells undergo apoptosis in order to avoid wasting nutrients and by their death to release nutrients for the benefit of younger and fitter cells. Thus, survival of the population is promoted, ${ }^{10}$ and under certain circumstances regrowth of fitter mutants is enabled. ${ }^{9}$ Importantly, chronological life span can be extended by activation of a stress response that involves specific transcription factors such as Msn2p or Msn4p, and transactivation of stress-response genes such as superoxide dismutase-2 (SOD2). ${ }^{55}$

Although both models of aging are clearly different and rely on partly opposing mechanisms, they seem to be coupled to some extent. Thus, the replicative life span of chronologically aged mother cells decreases with age. ${ }^{56}$ In fact, both models of aging depend on the nutritional status of the cell, which affects three nutrient-dependent kinases, TOR1/2, SCH9 (Akt/PKB), and protein kinase-A (PKA) ${ }^{56}$ Of note, hyperactivation of the Ras protein Ras $2 p$, which is part of a signaling pathway upstream from PKA activation, increases apoptosis while shortening the replicative and chronological life spans. ${ }^{57}$ This signaling pathway, which requires functional mitochondria for its execution, can be activated by mutations that reduce actin dynamics or enhance actin aggregation. ${ }^{38}$ In agreement with the observation that an increase in activity of nutrient-dependent kinases accelerates the aging process, nutrient-poor conditions result in life span extension, mimicking the longevity-associated effect of calorie restriction in mammals. Intriguingly, external supplementation of the polyamine spermidine promotes survival during aging in diverse organisms (yeast, with which the study was initiated, but also flies, worms, and human immune cells). In yeast, epigenetic deacetylation of histone $\mathrm{H} 3$ results in inhibition of a programmed type of necrosis (see Box 1) and induction of autophagy, a degradative and here clearly cytoprotective process. $^{7}$ Both mechanisms generally seem to play a significant role during aging.

Autophagy is induced by shortage of nutrients and can be viewed as a cellular pro-survival response of 'self-recycling' that plays a critical role in diverse human diseases. ${ }^{58}$
Macroautophagy leads to autophagosome formation, bulk vacuolar degradation of cytoplasmic organelles or protein aggregates, and subsequent release of amino acids and lipids into the cytosol. The phylogenetically conserved autophagyrelated genes (ATGs) are essential for autophagy in yeast. In addition, several autophagy pathways for specific protein and organelle degradation exist. Upstream from autophagic execution, nutrient availability is sensed via the TORC1 complex and cooperatively by Sch9p and PKA. ${ }^{59}$ Intriguingly, Whi2p, which is known to affect cell-cycle exit under nutritional stress conditions, is involved in the regulation of nutritional sensing and PKA signaling, where it plays a role in the differentiation of yeast colonies into distinct cell death zones. $^{60}$

Although autophagy mostly serves as a mechanism of cellular adaptation and survival, under specific circumstances it might mediate a certain type of PCD, defined as autophagic cell death. ${ }^{61}$ Cell death and autophagy, therefore, represent two distinct stress responses that might act in a cooperative or competitive manner depending on the cellular context. To what extent autophagy might as well bear a cell death function in yeast, remains to be explored. A possible candidate to be involved in such a process could be Uth1p, a mitochondrial protein required for mitophagy but also for death triggered by heterologous expression of human Bax. ${ }^{62}$ Deletion of UTH1 provides resistance to rapamycin and prevents ROS accumulation, but does not inhibit Bax insertion into the mitochondrial outer membrane or cytochrome $c$ release. ${ }^{62}$ Uth $1 p$ might, indeed, provide a direct association between cell death and mitochondrial turnover regulation via mitophagy.

\section{Proteins and Pathways Regulating Yeast Apoptosis}

The regulation of a death process such as yeast apoptosis requires complex interplay between small molecules, proteins, and pathways, which exhibit their functions at different locations in the cell, including the nucleus, mitochondria, the cytosol, or lysosomes. Figure 3 summarizes the interconnection of the different players involved in yeast apoptosis (Figure 3).

Small signaling molecules: ROS, NO, and ammonia. Since their first description as mediators of yeast apoptosis, ${ }^{21}$ ROS have been widely recognized as crucial cell death regulators and have been connected to many of the known apoptotic pathways in yeast (Figure 3). Potential cellular sources of ROS include the mitochondrial respiratory chain, the endoplasmic reticulum (ER), as well as the iron-coupled Fenton and HaberWeiss reactions. A further small molecule that is involved in yeast apoptosis is nitric oxide (NO), which is produced via an arginine-dependent mechanism in $\mathrm{H}_{2} \mathrm{O}_{2}$-induced apoptotic cells and $S$-nitrosates glyceraldehyde-3-phosphate dehydrogenase (GAPDH). Inhibition of NO synthesis promotes survival by decreasing both GAPDH S-nitrosation and intracellular ROS accumulation. ${ }^{63}$ During chronological aging, scavenging of NO by oxyhemoglobin delays the onset of death and reduces the levels of superoxide anion. ${ }^{63}$ Interestingly, the NO-target GAPDH has been implicated both in $\mathrm{H}_{2} \mathrm{O}_{2}$-induced yeast apoptosis $^{64}$ and in the regulation of apoptosis in human cells. $^{65}$ Finally, during development of multicellular yeast 


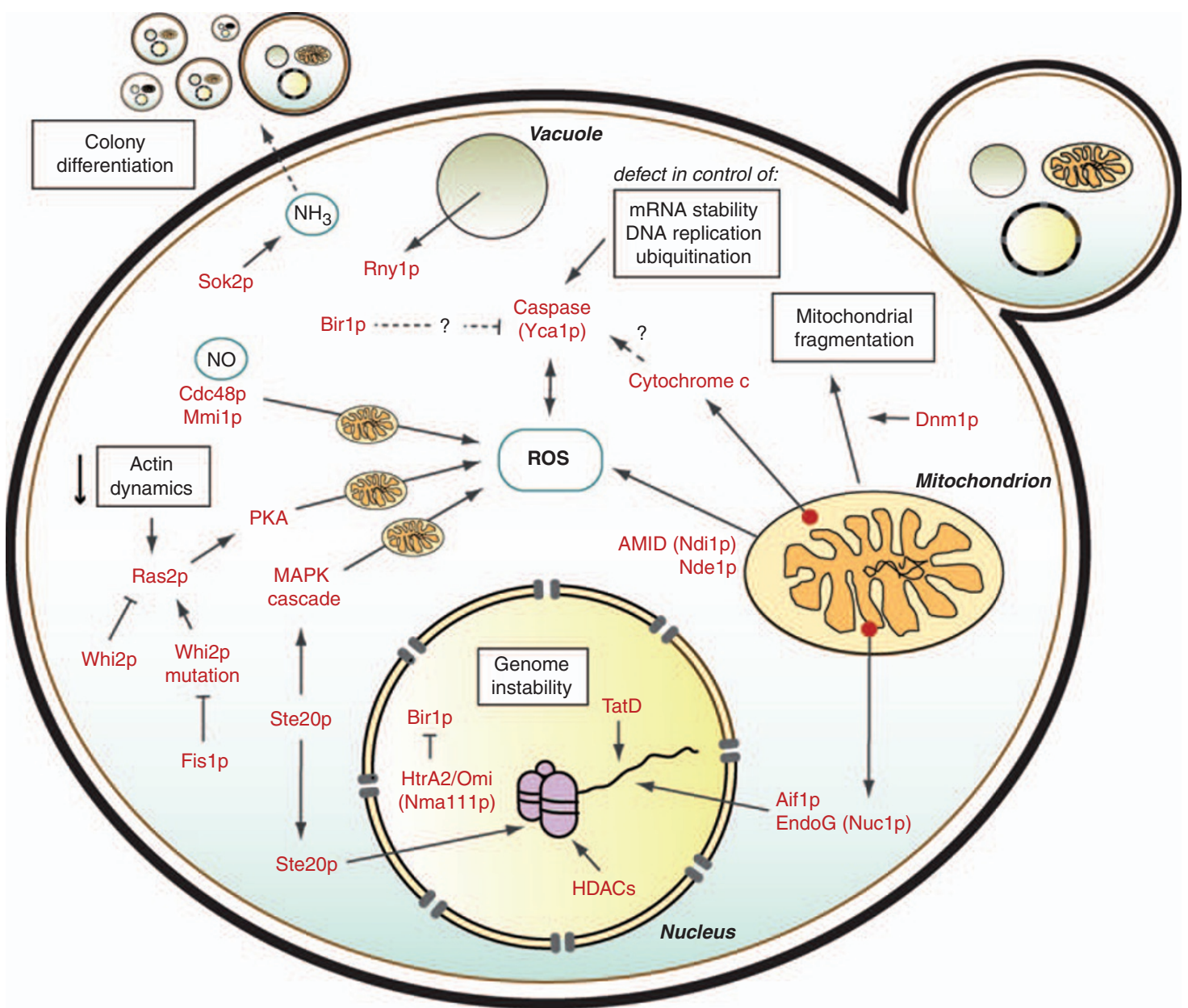

Figure 3 Proteins and pathways in yeast apoptosis. The network of factors involved in yeast apoptosis comprises several small molecules as lethal mediators, as well as an increasing number of orthologs of crucial mammalian apoptotic proteins that play a role in diverse conserved proteasomal, mitochondrial, vacuolar, and epigenetically regulated cell death pathways

colonies, ammonia accumulates in the center of the colonies and triggers the death of older cells, allowing young cells on the rim to exploit the released nutrients. Consistently, lack of the transcription factor Sok2p, which results in the inability to produce ammonia, leads to diffuse death throughout the whole population and diminishes the life span of the colony. ${ }^{4}$

The proteasome and the yeast caspase. The first molecular characterization of yeast apoptosis emerged from experiments performed using a CDC48 mutant $\left(c d c 48^{S 565 G}\right),{ }^{1}$ linking this death to proteomic alterations in mitochondria, release of cytochrome $c$ to the cytosol, increased ROS production, and development of apoptotic markers. Subsequently, the homologs of CDC48 in mammals (p97/VCP) and in $C$. elegans (mac-1) have been characterized as antiapoptotic factors. ${ }^{66}$ VCP is involved in ER-associated protein degradation (ERAD) and polyglutamine-induced neurodegeneration. ${ }^{66}$ Interestingly, impaired ERAD due to deletion of ERV29 in yeast causes ER stress and induction of the unfolded protein response (UPR), which results in ROS production by mitochondria and ER, and subsequent apoptosis. ${ }^{67}$
Yeast bears at least one ortholog of mammalian caspases: the metacaspase Yca1p. ${ }^{22}$ Numerous cell death scenarios have been shown to depend on Yca1p. This applies to oxygen stress, where disruption of YCA1 results in reduced cell death and decreased formation of apoptotic markers. ${ }^{22}$ Yca1p is also required for valproic acid-induced apoptosis, as well as for cell death following loss of ubiquitination control, altered mRNA stability, and defective initiation of DNA replication. 2,50,52 During chronological aging, deletion of YCA1 results in the accumulation of damaged cells due to the absence of the apoptotic cleaning function in the culture. In a direct competition assay, YCA1-mutant cells are actually outlived by wild-type cells when aged, ${ }^{10}$ an observation backed by the finding that YCA1 disruption increases the level of oxidized proteins. ${ }^{68}$ Altogether, these data underline the role of $Y C A 1$ in particular and metacaspases in general as pivotal players during cell death execution. This, in addition to the common evolutionary origin of caspases, argues for a functional conservation between metacaspases and caspases. Still, their different cleavage specificity - caspases cleave their substrates after aspartate (an acidic residue), metacaspases after arginine or lysine (basic residues) - has 
maintained skepticism concerning their molecular correspondence. In a recent study, however, Sundstrom et al. ${ }^{69}$ have uncovered the phylogenetically conserved protein TSN (Tudor staphylococcal nuclease) as the first common biological substrate of metacaspases and caspases. Their findings establish that, beyond phylogenetic distance and differences in their proteolytic characteristics, caspases and metacaspases do share natural, death-related target molecules, thus indicating that metacaspases and caspases indeed constitute functional homologs.

Yeast apoptosis can also occur independently of $Y C A 1 ;{ }^{35}$ for instance, during long-term development of yeast multicellular colonies, ${ }^{4}$ or when induced by defective $\mathrm{N}$-glycosylation in cells lacking Ost2p, the yeast homolog of the mammalian defender of apoptosis-1 (DAD1) protein. ${ }^{49}$ Moreover, apoptotic death mediated by the yeast apoptosis-inducing factor Aif1p or Nuc1p, the yeast homolog of endonuclease-G, does not require YCA1 ${ }^{5,6}$ Even though existence of caspase-like activities other than that of Yca1p cannot be excluded, these findings suggest that in yeast, as in mammals, apoptosis is not synonymous to caspase activity. ${ }^{35}$ Furthermore, the functional scope of Yca1p has been widened outside of the realm of apoptosis by a recent report implicating Yca1p in the regulation of the cell cycle. ${ }^{70}$ Coupling a vital and a lethal activity in one factor seems to be an element implanted in most if not all pro-death players. It is possible that a change in localization (as is the case for mitochondrial effectors such as Aif1p or Nuc1p) or a specific molecular alteration (which could apply to Yca1p abrogating its vital role) may allow the functional switch from vital to lethal. The advantage of such dual function is obvious: activation of the prodeath function connected to simultaneous disruption of the vital role cooperatively contributes to efficient execution of cellular demise and impedes the easy loss of any pro-death factor.

Nuclear factors. Nma111p (nuclear mediator of apoptosis), the yeast homolog of the proapoptotic mammalian HtrA2/ Omi, is another protease that might be involved in yeast apoptosis. While NMA111 deletion reduces apoptotic markers, its overexpression promotes cell death upon hyperthermia or $\mathrm{H}_{2} \mathrm{O}_{2}$ treatment. $^{71}$ In contrast to its human counterpart, which is localized in mitochondria, Nma111p resides in the nucleus and kills cells exclusively via its serine protease activity. ${ }^{71}$ Intriguingly, Nma111p can cleave the cytoplasm/nucleus-located Bir1p, the only known inhibitor-ofapoptosis (IAP) protein in yeast. ${ }^{72}$ After oxidative stress, $B I R 1$ disruption results in higher death rates. Consistently, overexpression of Bir1p reduces apoptosis, an effect that can be antagonized by simultaneous overexpression of $\mathrm{Nma}-$ $111 p$. Bir1p overexpression also delays the onset of cell death during chronological aging. ${ }^{72}$ Along with its function in apoptosis, Bir1p, like its closest animal homologs deterin (flies) and survivin (mammals), is involved in chromosome segregation and cytokinesis. Nma111p is not the only nuclear factor that has been implied in apoptotic regulation. Sequence comparison with apoptotic nucleases from $C$. elegans led to identification of the yeast dsDNA endo-/ exonuclease Tat- $D$. Under mild $\mathrm{H}_{2} \mathrm{O}_{2}$ stress conditions, Tat$D$ disruptants display better survival compared with wild-type controls, whereas overexpression of the nuclease leads to enhanced apoptosis. ${ }^{73}$
Another nuclear process that contributes to eukaryotic cell death control is histone $\mathrm{H} 2 \mathrm{~B}$ phosphorylation and, thus, epigenetic regulation. In yeast, the histone $\mathrm{H} 2 \mathrm{~B}$ tail $(\mathrm{H} 2 \mathrm{Bt})$ harbors a unidirectional cross-talk between Hos3p-directed lysine-11 (K11) deacetylation and subsequent serine-10 (S10) phosphorylation by the Ste20p kinase. A non-deacetylable $\mathrm{H} 2 \mathrm{Bt}$ lysine mutant elicited resistance against $\mathrm{H}_{2} \mathrm{O}_{2}-$ induced cell death, whereas a mutant that mimics a permanent deacetylated state strongly induced death. ${ }^{74}$ Besides Hos3p, two additional HDACs (Rpd3p and Hda1p) mediate cell death independently of $\mathrm{H} 2 \mathrm{Bt}$ deacetylation ${ }^{74}$ and antagonize the pro-survival effect of the H2B-acetylating enzyme Gcn5p during replicative aging. ${ }^{75}$ In addition, the kinase Ste20p has been shown to be necessary for pheromone-induced apoptosis in yeast. ${ }^{12}$ Therefore, H2B epigenetics may constitute a functional link between replicative life-span control and the pheromone-associated MAPK cascade. $^{75}$

Mitochondrial factors: friends when in and foes when out. In mammals, apoptosis-inducing factor (AIF), a flavoprotein with NADH oxidase activity normally contained in the mitochondrial intermembrane space, can contribute to apoptosis as a key mediator of caspase-independent cell death. The existence of an AIF homolog in yeast ${ }^{6}$ argues for the phylogenetic conservation of caspase-independent apoptosis pathways. Similar to its mammalian equivalent, yeast Aif1p undergoes a mitochondrial-nuclear shuttling upon apoptosis induction (via $\mathrm{H}_{2} \mathrm{O}_{2}$, acetate, or aging). Killing of yeast cells by Aif1p is dependent on yeast cyclophilin-A, comparable to the situation in mammals. ${ }^{6}$ The AIF1 deletion mutant exhibits improved survival following $\mathrm{H}_{2} \mathrm{O}_{2}$ and acetate treatment as well as during aging. ${ }^{6}$ Besides its lethal role, AIF also bears a vital function during optimal oxidative phosphorylation, which has been shown for both mammals and yeast. ${ }^{76}$

The NADH dehydrogenase Ndi1p, which catalyzes the oxidation of intramitochondrial NADH and is localized to the inner mitochondrial membrane, represents another mitochondrion-associated protein implicated in yeast cell death. ${ }^{77}$ Of note, its human homolog, the AIF-homologous, mitochondrion-associated inducer of cell death (AMID), has been linked to caspase-independent apoptosis. ${ }^{77}$ Disruption of NDI1 reduces ROS production and extends the chronological life span. Although to a lower extent, knockout of NDE1, located on the outer mitochondrial membrane and responsible for oxidation of cytosolic $\mathrm{NADH}$, does also exhibit this antiaging effect. ${ }^{77}$

Cytochrome $c$ is a mitochondrial protein with a genuine function in the respiratory chain and, in mammals, additionally a well-characterized lethal factor implicated in the activation of caspase- 9 in the intrinsic pathway of apoptosis. Although several studies have connected cytochrome $c$ release with yeast apoptosis, ${ }^{26,27}$ it remains elusive whether cytochrome $c$ can lead to the formation of an apoptosome-like structure and subsequent caspase activation, as it occurs in mammalian cells. Until now, neither homologs of the mammalian apoptosome-associated factor Apaf nor functionally related proteins have been identified in yeast. In fact, to date the involvement of cytochrome $c$ in the execution of yeast cell 
death has not been completely clarified. Activation of caspase function, in analogy to the mammalian system, is a possibility that could involve either Yca1p or another factor exhibiting caspase activity yet to be identified. However, cytochrome $c$ could also represent an ancestral pro-death factor, whose lethal role could only rely on the indirect consequence of respiratory dysfunction and subsequent ROS accumulation upon release into the cytosol. In this case, it would not intervene in direct execution - this would have been developed later in evolution.

Nuc1p, the yeast ortholog of the proapoptotic endonuclease-G (EndoG), is a further cell death-inducing factor that is located in mitochondria. ${ }^{5}$ Similar to its mammalian counterpart, Nuc1p translocates to the nucleus upon apoptosis induction. Consistently, apoptosis can be efficiently triggered by a Nuc1p variant, which lacks the mitochondrial localization sequence. Nuc1p-triggered death is reduced in yeast cells that lack the homologs of the mammalian adenine translocator, thus arguing for the implication of the PTP in yeast cell death. ${ }^{5}$ In addition, karyopherin Kap123p and histone H2B (but not Yca1p and Aif1p) are necessary for Nuc1p-mediated apoptosis. ${ }^{5}$ Similar to other apoptotic players, Nuc1p exerts lethal and vital functions as illustrated by deletion of the gene, which inhibits apoptosis when mitochondrial respiration is enhanced, but increases necrotic death when oxidative phosphorylation is repressed..$^{5}$ In fact, a vital role of EndoG in the maintenance of polyploid cells has been recently described for both yeast cells and human cancer cells. ${ }^{78}$

In humans, mitochondrial fragmentation (fission) plays a causal role in apoptosis and is regulated by the dynaminrelated protein-1 (Drp1). Mitochondrial shape transitions are critical for yeast cell death, as well: for instance, upon challenge with diverse death stimuli, the yeast homolog of Drp1 (Dnm1p) promotes apoptosis preceded by mitochondrial fragmentation and degradation. ${ }^{25}$ In turn, deletion of DNM1 and consequently reduced mitochondrial fission has been shown to elongate the life span. ${ }^{79}$ Two interactors of Dnm1p with a fission function in healthy cells are also involved in cell death regulation: (i) Mdv1p/Net2p consistently promotes cell death, whereas (ii) Fis1p unexpectedly appears to have a prosurvival effect, as suggested by a YCA1-dependent increase in the death rate of FIS1-deficient cells. ${ }^{25}$ However, a recent report has clarified that deletion of FIS1 actually selects for a secondary mutation in the stress-response gene WHI2 that confers sensitivity to cell death. ${ }^{80}$ This finding actually emphasizes the issue of secondary mutations and proper application of the yeast knockout collection. It seems advisable to take this issue into consideration when interpreting phenotypic results, for example, upon epistatic analyses using the yeast knockout collection, and to pursue the identification of such cryptic mutations. In fact, such mutations may well lead to informative new genetic interactions. Still, the mitochondrial fission death pathway is involved in several apoptotic scenarios, including exposure to amiodarone or mating factor, where mitochondrial fragmentation is dependent on Ysp1p, M1 virus-encoded K1 toxin, or ethanol. ${ }^{2}$

Finally, Mmi1p, the yeast ortholog of human TCTP (translationally controlled tumor protein), which is involved in apoptosis, translocates to mitochondria upon oxidative stress. Deletion of MMI1 increases both resistance to $\mathrm{H}_{2} \mathrm{O}_{2}$ and life span during replicative aging. Interestingly, MMI1 disruptants show benomyl sensitivity, pointing toward a role of the protein in stabilizing microtubules and, thus, establishing a functional link between the cytoskeleton and mitochondria. ${ }^{81}$

Yeast lysosomes and peroxisomes. In addition to mitochondria, lysosomes have been shown to play an active role in mammalian PCD regulation. On lethal insult, hydrolases are released from the lysosomal lumen into the cytosol following lysosomal membrane permeabilization (LMP). ${ }^{82}$ A recent study reveals that yeast lysosomes (vacuoles) are also directly involved in cell death. ${ }^{83}$ After oxidative stress, the RNase T2-family member Rny1p is released from the vacuole into the cytosol and then directly promotes cell death independently of its catalytic activity. To what extent other vacuolar factors participate in yeast cell death regulation, remains to be explored. Vacuolar hydrolases are among the possible candidates. In fact, Pep4p (the homolog of mammalian cathepsin-D) has been shown to migrate out of the vacuoles and degrade nucleoporins upon $\mathrm{H}_{2} \mathrm{O}_{2}$-induced cell death. ${ }^{84}$ However, the associated form(s) of death remains to be accurately characterized.

Furthermore, peroxisomal function, linked to hydrogen peroxide metabolism, may also be directly connected to cell death pathways in yeast. For instance, deletion of PEX6, coding for a protein essential for peroxisomal protein import, induced necrotic cell death upon acetic acid treatment and upon entry into the early stationary phase. ${ }^{85}$ To what extent this necrotic death might be programmed, needs to be further elucidated. Furthermore, pexophagy (selective degradation of peroxisomes) represents a direct connection to the process of autophagy. The mechanistic significance of pexophagy for autophagic cell death or other forms of cell death will need to be clarified. It is interesting, however, that the cytoskeleton has been described to be involved in pexophagy, ${ }^{86}$ which invites the speculation that peroxisome function and degradation might cooperate with actin dynamics, which is a crucial process for apoptosis. ${ }^{87}$

\section{Conclusion}

One of the biggest challenges in modern medicine is the comprehension of PCD in the context of cancer and autoimmune, cardiovascular, as well as neurodegenerative diseases. Accumulating evidence points toward the phylogenetic conservation of the core machinery and the core regulators of cell death between yeast and mammals. This has opened up the possibility to use yeast as a research tool that can provide new clues in the elucidation of cell death pathways in higher eukaryotes. In fact, the yeast apoptotic model has already demonstrated its potential for application in the human system, as exemplified by the increasing number of studies using yeast as a model for neurotoxicity and cancer. ${ }^{47,88}$ Importantly, several mammalian counterparts of yeast cell death factors play major roles in the pathogenesis of disease. Among them are the caspases, involved in many physiological and pathological states; AIF, whose absence can cause neurodegeneration, skeleton muscle atrophy, and dilated cardiomyopathy; or endonuclease-G, whose nuclear translocation has been associated with cerebral ischemia and 
muscular atrophy. Yeast also serves as a model for aging of post-mitotic cells (chronological aging) as well as that of stem cells (replicative aging), and has shed light on the possibility of fighting unicellular fungal or protozoan pathogens by the pharmacological induction of PCD. Of note, yeast represents a eukaryote with easily manipulable mitochondria, which are emerging as central organelles in the regulation of apoptosis.

It is tempting to speculate that yeast cell death may not only be regulated by its apoptotic machinery but also via other types of PCD mechanisms. As a simple and technically advantageous eukaryotic model organism, yeast might, therefore, help to uncover the pathophysiological and mechanistic implications of 'universal' activators, inhibitors, and executors of PCD, including the connection of PCDregulatory networks to cell-cycle control, mitochondrial metabolism, lipotoxic processes, and epigenetics. Yeast might also help to understand the switches that determine to which particular PCD subroutine (e.g. apoptosis, autophagy, necrosis) cells succumb. It is as important to learn how to live as to know how to die - at least in the context of pathologyoriented cell biology, yeast will teach us invaluable lessons on the mysteries of health and disease.

\section{Conflict of interest}

The authors declare no conflict of interest.

Acknowledgements. We are grateful to the Austrian Science Fund FWF (Austria) for grant S-9304-B05 to FM, SB, and DC-G; grant 'SFB Lipotox' to FM and DC-G; grant T-414-B09 to SB (Hertha-Firnberg fellowship); and to the European Commission for project APOSYS to FM and TE. CM is supported by the Excellence Initiative of the German Federal \& State Governments (EXC 294) and the Trinational Research Training Group (GRK 1478); GK is supported by the Ligue Nationale contre le Cancer (Equipe labellisée), Agence Nationale pour la Recherche (ANR), European Commission (Apo-Sys, ChemoRes, ApopTrain), Fondation pour la Recherche Médicale (FRM), Institut National du Cancer (INCa), and Cancéropôle lle-de-France.

1. Madeo $F$, Frohlich $E$, Frohlich KU. A yeast mutant showing diagnostic markers of early and late apoptosis. J Cell Biol 1997; 139: 729-734.

2. Carmona-Gutierrez D, Madeo F. Tracing the roots of death: apoptosis in Saccharomyces cerevisiae. In: Dong Z, Yin X-M (eds). Essentials of Apoptosis - A Guide for Basic and Clinical Research. Humana Press, 2009, pp 325-354.

3. Ribeiro GF, Corte-Real M, Johansson B. Characterization of DNA damage in yeast apoptosis induced by hydrogen peroxide, acetic acid, and hyperosmotic shock. $\mathrm{Mol} B \mathrm{BiO}$ Cell 2006; 17: 4584-4591.

4. Vachova L, Palkova Z. Physiological regulation of yeast cell death in multicellular colonies is triggered by ammonia. J Cell Biol 2005; 169: 711-717.

5. Buttner S, Eisenberg T, Carmona-Gutierrez D, Ruli D, Knauer $\mathrm{H}$, Ruckenstuhl $\mathrm{C}$ et al. Endonuclease $G$ regulates budding yeast life and death. Mol Cell 2007; 25: 233-246.

6. Wissing S, Ludovico P, Herker E, Buttner S, Engelhardt SM, Decker T et al. An AIF orthologue regulates apoptosis in yeast. J Cell Biol 2004; 166: 969-974.

7. Eisenberg T, Knauer H, Schauer A, Buttner S, Ruckenstuhl C, Carmona-Gutierrez D et al. Induction of autophagy by spermidine promotes longevity. Nat Cell Biol 2009; 11: 1305-1314.

8. Buttner S, Eisenberg T, Herker E, Carmona-Gutierrez D, Kroemer G, Madeo F. Why yeast cells can undergo apoptosis: death in times of peace, love, and war. J Cell Biol 2006; 175 521-525.

9. Fabrizio P, Battistella L, Vardavas R, Gattazzo C, Liou LL, Diaspro A et al. Superoxide is a mediator of an altruistic aging program in Saccharomyces cerevisiae. J Cell Biol 2004; 166 1055-1067.

10. Herker $\mathrm{E}$, Jungwirth $\mathrm{H}$, Lehmann KA, Maldener $\mathrm{C}$, Frohlich $\mathrm{KU}$, Wissing $\mathrm{S}$ et al. Chronological aging leads to apoptosis in yeast. J Cell Biol 2004; 164: 501-507.

11. Laun P, Pichova A, Madeo F, Fuchs J, Ellinger A, Kohlwein S et al. Aged mother cells of Saccharomyces cerevisiae show markers of oxidative stress and apoptosis. Mol Microbiol 2001; 39: 1166-1173.
12. Severin FF, Hyman AA. Pheromone induces programmed cell death in $S$. cerevisiae Curr Biol 2002; 12: R233-R235.

13. Ahn SH, Henderson KA, Keeney S, Allis CD. H2B (Ser10) phosphorylation is induced during apoptosis and meiosis in S. cerevisiae. Cell Cycle 2005; 4: 780-783.

14. Knorre DA, Smirnova EA, Severin FF. Natural conditions inducing programmed cell death in the yeast Saccharomyces cerevisiae. Biochemistry (Moscow) 2005; 70: 264-266.

15. Yuan JY, Horvitz HR. The Caenorhabditis elegans genes ced-3 and ced-4 act cell autonomously to cause programmed cell death. Dev Biol 1990; 138: 33-41.

16. Lam E. Controlled cell death, plant survival and development. Nat Rev Mol Cell Biol 2004; 5: 305-315.

17. Ameisen JC. Looking for death at the core of life in the light of evolution. Cell Death Differ 2004; 11: 4-10

18. Reiter J, Herker E, Madeo F, Schmitt MJ. Viral killer toxins induce caspase-mediated apoptosis in yeast. J Cell Biol 2005; 168: 353-358.

19. Morton CO, Dos Santos SC, Coote P. An amphibian-derived, cationic, alpha-helical antimicrobial peptide kills yeast by caspase-independent but AlF-dependent programmed cell death. Mol Microbiol 2007; 65: 494-507.

20. Narasimhan ML, Coca MA, Jin J, Yamauchi T, Ito $Y$, Kadowaki T et al. Osmotin is a homolog of mammalian adiponectin and controls apoptosis in yeast through a homolog of mammalian adiponectin receptor. Mol Cell 2005; 17: 171-180.

21. Madeo F, Frohlich E, Ligr M, Grey M, Sigrist SJ, Wolf DH et al. Oxygen stress: a regulator of apoptosis in yeast. J Cell Biol 1999; 145: 757-767.

22. Madeo F, Herker E, Maldener C, Wissing S, Lachelt S, Herlan M et al. A caspase-related protease regulates apoptosis in yeast. Mol Cell 2002; 9: 911-917.

23. Singh K, Kang PJ, Park HO. The Rho5 GTPase is necessary for oxidant-induced cell death in budding yeast. Proc Natl Acad Sci USA 2008; 105: 1522-1527.

24. Ludovico P, Sousa MJ, Silva MT, Leao C, Corte-Real M. Saccharomyces cerevisiae commits to a programmed cell death process in response to acetic acid. Microbiology 2001; 147 (Pt 9): 2409-2415.

25. Fannjiang Y, Cheng WC, Lee SJ, Qi B, Pevsner J, McCaffery JM et al. Mitochondrial fission proteins regulate programmed cell death in yeast. Genes Dev 2004; 18 2785-2797.

26. Ludovico P, Rodrigues F, Almeida A, Silva MT, Barrientos A, Corte-Real M. Cytochrome c release and mitochondria involvement in programmed cell death induced by acetic acid in Saccharomyces cerevisiae. Mol Biol Cell 2002; 13: 2598-2606.

27. Pereira C, Camougrand N, Manon S, Sousa MJ, Corte-Real M. ADP/ATP carrier is required for mitochondrial outer membrane permeabilization and cytochrome $c$ release in yeast apoptosis. Mol Microbiol 2007; 66: 571-582.

28. Valenti D, Vacca RA, Guaragnella N, Passarella S, Marra E, Giannattasio S. A transient proteasome activation is needed for acetic acid-induced programmed cell death to occur in Saccharomyces cerevisiae. FEMS Yeast Res 2008; 8: 400-404

29. Burtner CR, Murakami CJ, Kennedy BK, Kaeberlein M. A molecular mechanism of chronological aging in yeast. Cell Cycle 2009; 8: 1256-1270.

30. Almeida B, Ohlmeier S, Almeida AJ, Madeo F, Leao C, Rodrigues F et al. Yeast protein expression profile during acetic acid-induced apoptosis indicates causal involvement of the TOR pathway. Proteomics 2009; 9: 720-732.

31. Almeida T, Marques M, Mojzita D, Amorim MA, Silva RD, Almeida B et al. Isc1p plays a key role in hydrogen peroxide resistance and chronological lifespan through modulation of iron levels and apoptosis. Mol Biol Cell 2008; 19: 865-876.

32. Aerts AM, Zabrocki P, Francois IE, Carmona-Gutierrez D, Govaert G, Mao C et al. Ydc1p ceramidase triggers organelle fragmentation, apoptosis and accelerated ageing in yeast. Cell Mol Life Sci 2008; 65: 1933-1942.

33. Low CP, Shui G, Liew LP, Buttner S, Madeo F, Dawes IW et al. Caspase-dependent and -independent lipotoxic cell-death pathways in fission yeast. J Cell Sci 2008; 121 (Pt 16): 2671-2684

34. Garbarino J, Padamsee M, Wilcox L, Oelkers PM, D'Ambrosio D, Ruggles KV et al. Sterol and diacylglycerol acyltransferase deficiency triggers fatty acid-mediated cell death. J Biol Chem 2009; 284: 30994-31005

35. Madeo F, Carmona-Gutierrez D, Ring J, Buttner S, Eisenberg T, Kroemer G. Caspasedependent and caspase-independent cell death pathways in yeast. Biochem Biophys Res Commun 2009; 382: 227-231.

36. Pozniakovsky AI, Knorre DA, Markova OV, Hyman AA, Skulachev VP, Severin FF. Role of mitochondria in the pheromone- and amiodarone-induced programmed death of yeast. $J$ Cell Biol 2005; 168: 257-269.

37. Madeo F, Durchschlag M, Kepp O, Panaretakis T, Zitvogel L, Frohlich KU et al. Phylogenetic conservation of the preapoptotic calreticulin exposure pathway from yeast to mammals. Cell Cycle 2009; 8: 639-642.

38. Gourlay CW, Du W, Ayscough KR. Apoptosis in yeast - mechanisms and benefits to a unicellular organism. Mol Microbiol 2006; 62: 1515-1521.

39. Almeida B, Silva A, Mesquita A, Sampaio-Marques B, Rodrigues F, Ludovico P. Drug-induced apoptosis in yeast. Biochim Biophys Acta 2008; 1783: 1436-1448.

40. Sun $Q$, Bi L, Su X, Tsurugi K, Mitsui K. Valproate induces apoptosis by inducing accumulation of neutral lipids which was prevented by disruption of the SIR2 gene in Saccharomyces cerevisiae. FEBS Lett 2007; 581: 3991-3995.

41. Zhang YQ, Rao R. Global disruption of cell cycle progression and nutrient response by the antifungal agent amiodarone. J Biol Chem 2007; 282: 37844-37853. 
42. Zhang NN, Dudgeon DD, Paliwal S, Levchenko A, Grote E, Cunningham KW. Multiple signaling pathways regulate yeast cell death during the response to mating pheromones. Mol Biol Cell 2006; 17: 3409-3422.

43. Eisenberg T, Buttner S, Kroemer G, Madeo F. The mitochondrial pathway in yeast apoptosis. Apoptosis 2007; 12: 1011-1023.

44. Longo VD, Ellerby LM, Bredesen DE, Valentine JS, Gralla EB. Human Bcl-2 reverses survival defects in yeast lacking superoxide dismutase and delays death of wild-type yeast. J Cell Biol 1997; 137: 1581-1588.

45. Outeiro TF, Lindquist S. Yeast cells provide insight into alpha-synuclein biology and pathobiology. Science 2003; 302: 1772-1775.

46. Buttner S, Bitto A, Ring J, Augsten M, Zabrocki P, Eisenberg T et al. Functional mitochondria are required for alpha-synuclein toxicity in aging yeast. J Biol Chem 2008; 283: $7554-7560$.

47. Braun RJ, Buttner S, Ring J, Kroemer G, Madeo F. Nervous yeast: modeling neurotoxic cell death. Trends Biochem Sci 2009; doi:10.1016/j.tibs.2009.10.005

48. Winderickx J, Delay C, De Vos A, Klinger H, Pellens K, Vanhelmont T et al. Protein folding diseases and neurodegeneration: lessons learned from yeast. Biochim Biophys Acta 2008; 1783: 1381-1395.

49. Hauptmann P, Riel C, Kunz-Schughart LA, Frohlich KU, Madeo F, Lehle L. Defects in $\mathrm{N}$-glycosylation induce apoptosis in yeast. Mol Microbiol 2006; 59: 765-778.

50. Mazzoni C, Herker E, Palermo V, Jungwirth H, Eisenberg T, Madeo F et al. Yeas caspase 1 links messenger RNA stability to apoptosis in yeast. EMBO Rep 2005; 6 : 1076-1081.

51. Yang $\mathrm{H}$, Ren $\mathrm{Q}$, Zhang Z. Cleavage of Mcd1 by caspase-like protease Esp1 promotes apoptosis in budding yeast. Mol Biol Cell 2008; 19: 2127-2134.

52. Weinberger M, Ramachandran L, Feng L, Sharma K, Sun X, Marchetti M et al. Apoptosis in budding yeast caused by defects in initiation of DNA replication. J Cell Sci 2005; 118 (Pt 15): 3543-3553.

53. Begley U, Dyavaiah M, Patil A, Rooney JP, DiRenzo D, Young CM et al. Trm9-catalyzed tRNA modifications link translation to the DNA damage response. Mol Cell 2007; 28 : 860-870.

54. Iraqui I, Kienda G, Soeur J, Faye G, Baldacci G, Kolodner RD et al. Peroxiredoxin Tsa1 is the key peroxidase suppressing genome instability and protecting against cell death in Saccharomyces cerevisiae. PLoS Genet 2009; 5: e1000524.

55. Fabrizio P, Liou LL, Moy VN, Diaspro A, Valentine JS, Gralla EB et al. SOD2 functions downstream of Sch9 to extend longevity in yeast. Genetics 2003; 163: 35-46.

56. Rockenfeller P, Madeo F. Apoptotic death of ageing yeast. Exp Gerontol 2008; 43: 876-881

57. Longo VD. Ras: the other pro-aging pathway. Sci Aging Knowledge Environ 2004; 2004 pe36.

58. Levine B, Kroemer G. Autophagy in the pathogenesis of disease. Cell 2008; 132: 27-42.

59. Yorimitsu T, Zaman S, Broach JR, Klionsky DJ. Protein kinase A and Sch9 cooperatively regulate induction of autophagy in Saccharomyces cerevisiae. Mol Biol Cell 2007; 18: 4180-4189.

60. Leadsham JE, Miller K, Ayscough KR, Colombo S, Martegani E, Sudbery P et al. Whi2p links nutritional sensing to actin-dependent Ras-cAMP-PKA regulation and apoptosis in yeast. J Cell Sci 2009; 122 (Pt 5): 706-715.

61. Kourtis N, Tavernarakis N. Autophagy and cell death in model organisms. Cell Death Differ 2009; 16: 21-30.

62. Camougrand N, Kissova I, Velours G, Manon S. Uth1p: a yeast mitochondrial protein at the crossroads of stress, degradation and cell death. FEMS Yeast Res 2004; 5: 133-140.

63. Almeida B, Buttner S, Ohlmeier S, Silva A, Mesquita A, Sampaio-Marques B et al NO-mediated apoptosis in yeast. J Cell Sci 2007; 120 (Pt 18): 3279-3288.

64. Magherini F, Tani C, Gamberi T, Caselli A, Bianchi L, Bini L et al. Protein expression profiles in Saccharomyces cerevisiae during apoptosis induced by $\mathrm{H}_{2} \mathrm{O}_{2}$. Proteomics 2007; 7: 1434-1445.

65. Tarze A, Deniaud A, Le Bras M, Maillier E, Molle D, Larochette N et al. GAPDH, a nove regulator of the pro-apoptotic mitochondrial membrane permeabilization. Oncogene 2007 26: 2606-2620.

66. Braun RJ, Zischka H. Mechanisms of Cdc48/VCP-mediated cell death: from yeast apoptosis to human disease. Biochim Biophys Acta 2008; 1783: 1418-1435.
67. Haynes CM, Titus EA, Cooper AA. Degradation of misfolded proteins prevents ER-derived oxidative stress and cell death. Mol Cell 2004; 15: 767-776.

68. Khan MA, Chock PB, Stadtman ER. Knockout of caspase-like gene, YCA1, abrogates apoptosis and elevates oxidized proteins in Saccharomyces cerevisiae. Proc Natl Acad Sci USA 2005; 102: 17326-17331.

69. Sundstrom JF, Vaculova A, Smertenko AP, Savenkov El, Golovko A, Minina E et al. Tudo staphylococcal nuclease is an evolutionarily conserved component of the programmed cell death degradome. Nat Cell Biol 2009; 11: 1347-1354.

70. Lee RE, Puente LG, Kaern M, Megeney LA. A non-death role of the yeast metacaspase: Yca1p alters cell cycle dynamics. PLoS One 2008; 3: e2956.

71. Fahrenkrog B, Sauder U, Aebi U. The S. cerevisiae HtrA-like protein Nma111p is a nuclear serine protease that mediates yeast apoptosis. J Cell Sci 2004; 117 (Pt 1): 115-126.

72. Walter D, Wissing S, Madeo F, Fahrenkrog B. The inhibitor-of-apoptosis protein Bir1p protects against apoptosis in $\mathrm{S}$. cerevisiae and is a substrate for the yeast homologue of Omi/HtrA2. J Cell Sci 2006; 119 (Pt 9): 1843-1851.

73. Qiu J, Yoon JH, Shen B. Search for apoptotic nucleases in yeast: role of Tat-D nuclease in apoptotic DNA degradation. J Biol Chem 2005; 280: 15370-15379.

74. Ahn SH, Diaz RL, Grunstein M, Allis CD. Histone H2B deacetylation at lysine 11 is required for yeast apoptosis induced by phosphorylation of $\mathrm{H} 2 \mathrm{~B}$ at serine 10. Mol Cell 2006; 24 : 211-220.

75. Carmona-Gutierrez D, Madeo F. Yeast unravels epigenetic apoptosis control: deadly chat within a histone tail. Mol Cell 2006; 24: 167-169.

76. Modjtahedi N, Giordanetto F, Madeo F, Kroemer G. Apoptosis-inducing factor: vital and lethal. Trends Cell Biol 2006; 16: 264-272.

77. Li W, Sun L, Liang Q, Wang J, Mo W, Zhou B. Yeast AMID homologue Ndi1p displays respiration-restricted apoptotic activity and is involved in chronological aging. $\mathrm{Mol} B \mathrm{Biol} \mathrm{Cell}$ 2006; 17: 1802-1811.

78. Buttner S, Carmona-Gutierrez D, Vitale I, Castedo M, Ruli D, Eisenberg T et al. Depletion of endonuclease G selectively kills polyploid cells. Cell Cycle 2007; 6: 1072-1076.

79. Scheckhuber CQ, Erjavec N, Tinazli A, Hamann A, Nystrom T, Osiewacz HD. Reducing mitochondrial fission results in increased life span and fitness of two fungal ageing models. Nat Cell Biol 2007; 9: 99-105.

80. Cheng WC, Teng X, Park HK, Tucker CM, Dunham MJ, Hardwick JM. Fis1 deficiency selects for compensatory mutations responsible for cell death and growth control defects. Cell Death Differ 2008; 15: 1838-1846.

81. Rinnerthaler M, Jarolim S, Heeren G, Palle E, Perju S, Klinger H et al. MMl1 (YKL056c, TMA19), the yeast orthologue of the translationally controlled tumor protein (TCTP) has apoptotic functions and interacts with both microtubules and mitochondria. Biochim Biophys Acta 2006; 1757: 631-638.

82. Boya P, Kroemer G. Lysosomal membrane permeabilization in cell death. Oncogene 2008; 27: 6434-6451.

83. Thompson DM, Parker R. The RNase Rny1p cleaves tRNAs and promotes cell death during oxidative stress in Saccharomyces cerevisiae. J Cell Biol 2009; 185: 43-50.

84. Mason DA, Shulga N, Undavai S, Ferrando-May E, Rexach MF, Goldfarb DS. Increased nuclear envelope permeability and Pep4p-dependent degradation of nucleoporins during hydrogen peroxide-induced cell death. FEMS Yeast Res 2005; 5: 1237-1251.

85. Jungwirth H, Ring J, Mayer T, Schauer A, Buttner S, Eisenberg T et al. Loss of peroxisome function triggers necrosis. FEBS Lett 2008; 582: 2882-2886.

86. Reggiori F, Monastyrska I, Shintani T, Klionsky DJ. The actin cytoskeleton is required for selective types of autophagy, but not nonspecific autophagy, in the yeast Saccharomyces cerevisiae. Mol Biol Cell 2005; 16: 5843-5856.

87. Gourlay CW, Carpp LN, Timpson P, Winder SJ, Ayscough KR. A role for the actin cytoskeleton in cell death and aging in yeast. J Cell Biol 2004; 164: 803-809.

88. Ruckenstuhl C, Buttner S, Carmona-Gutierrez D, Eisenberg T, Kroemer G, Sigrist SJ et al. The Warburg effect suppresses oxidative stress induced apoptosis in a yeast model for cancer. PLoS One 2009; 4: e4592.

89. Kroemer G, Galluzzi L, Vandenabeele P, Abrams J, Alnemri ES, Baehrecke EH et al. Classification of cell death: recommendations of the Nomenclature Committee on Cell Death 2009. Cell Death Differ 2009; 16: 3-11. 